\title{
Fatigue, sleep, and nocturnal complaints in patients with amyotrophic lateral sclerosis
}

\author{
D. Lo Coco ${ }^{a, b}$ and V. La Bella ${ }^{a}$ \\ ${ }^{\mathrm{a}}$ ALS Research Center, Dipartimento di Biomedicina Sperimentale e Neuroscienze Cliniche, Università di Palermo, Palermo; ${ }^{\mathrm{b}}$ Sleep \\ Disorders Clinic, U.O. Neurologia e Neurofisiopatologia, Dipartimento di Neuroscienze, Ospedale Civico - ARNAS, Palermo, Italy
}

\section{Keywords:}

ALS Functional Rating Scale-Revised, amyotrophic lateral sclerosis, cramps, fatigue, nocturia, sleep

Received 17 October 2011 Accepted 17 November 2011
Background and purpose: Fatigue is a common symptom in amyotrophic lateral sclerosis (ALS). Although sleep disturbances are a candidate factor that may interfere with fatigue in patients with ALS, the role of sleep-related abnormalities in determining fatigue in ALS is unknown.

Objective: To evaluate the frequency and determinants of fatigue in a group of 91 consecutive patients with ALS, with special attention to the relationship between fatigue and sleep problems.

Methods: Measures included the Fatigue Severity Scale (FSS), Pittsburgh Sleep Quality Index (PSQI), Epworth Sleepiness Scale (ESS), ALS Functional Rating ScaleRevised (ALSFRS-R), and Beck Depression Inventory (BDI).

Results: The mean FSS score was $4.35 \pm 1.1$, and 48 patients with ALS $(52.75 \%)$ reported clinical significant fatigue. FSS score correlated with ALSFRS-R score, forced vital capacity, ESS, BDI, and global PSQI score. Patients with fatigue were significantly more disabled and more frequently reported difficulties staying asleep and nocturnal complaints, such as nocturia and disturbing muscle cramps. After multivariate analysis, patients' disability and nocturnal complaints were significantly associated with fatigue.

Conclusion: In this study, we demonstrated that fatigue, a troublesome and disabling symptom in ALS, is associated with physical impairment and night-time complaints (such as nocturia and muscle cramps), suggesting that treating sleep problems might be useful in alleviating fatigue in these patients.

\section{Introduction}

Fatigue is a common symptom in amyotrophic lateral sclerosis (ALS), affecting $44-86 \%$ of patients with ALS [1-3]. Fatigue has been defined as an overwhelming sense of tiredness, lack of energy, and feeling of exhaustion [4]. It has an important impact on quality of life in patients with ALS [1], and it has been indicated as one of the reasons for shortening life in a Dutch study [5]. Fatigue in ALS has been associated with disease progression, age, site of ALS onset, ALS severity, and depression [1-3].

The causes and mechanisms of fatigue in ALS are multifactorial, including both central and peripheral fatigue [4]. Psychological factors linked to disease evolution and the physical obligates of daily life, respira-

Correspondence: Daniele Lo Coco, ALS Research Center, Dipartimento di Biomedicina Sperimentale e Neuroscienze Cliniche (BioNeC), Università di Palermo, Via G. La Loggia, 1 - 90129 Palermo, Italy (tel.: + 39091655 5158; fax: + 39091655 5198; e-mail: danielelococo@yahoo.com). tory impairment, and medications (including riluzole, antispasticity, and antidepressants) could also play a role [4].

Poor quality of sleep and excessive daytime somnolence could also contribute to fatigue in ALS. In previous studies, patients with ALS showed reduced total sleep time, reduced sleep efficiency, and increased apneas and hypopneas [6,7]. Moreover, patients with ALS frequently reported night-time complaints such as nocturia, muscle cramps, and restless legs syndrome [79]. However, whether specific aspects of sleep affect fatigue in patients with ALS remains unknown [4].

Therefore, we decided to investigate the frequency and determinants of fatigue in a large group of patients with ALS, with special attention to the relationship between fatigue and sleep problems.

\section{Patients and methods}

We collected detailed clinical data of 91 consecutive patients with ALS during their routine visit to our ALS 
Center between February 2009 and June 2011. Patients were diagnosed as definite or probable ALS according to the El-Escorial WFN revised criteria [10].

All subjects underwent a standardized face-to-face interview with a neurologist and expert in sleep medicine (DLC), board certified by the Italian Association of Sleep Medicine. The structured interview collected data on sleep problems ('yes' or 'no' type of answer). Subjects had to specify whether they had: (i) persistent initial insomnia (difficulty falling asleep within $30 \mathrm{~min}$ or more); (ii) difficulties staying asleep (more than two $\geq 30$-min nocturnal awakenings independently of the need to use the bathroom, or undesirable early morning awakening with inability to fall asleep again); (iii) unrefreshing sleep (feeling tired and sleepy after waking in the morning). Symptoms should have lasted at least for 1 month and had an impact on the subject's daily life. Information was also sought on whether patients snored, had nocturia, postural discomfort, pain, or muscle cramps. Nocturnal complaints were defined by the presence of two or more of the above-mentioned symptoms for at least three nights at week, and they had to cause subjective discomfort.

In addition, the Pittsburgh Sleep Quality Index (PSQI) was administered to all the study participants [11]. A PSQI score $>5$ was taken to indicate poor sleep. Daytime somnolence was evaluated using the Epworth Sleepiness Scale (ESS) [12]. Excessive daytime sleepiness (EDS) was defined by an ESS score $\geq 10$.

Fatigue was evaluated by the Fatigue Severity Scale (FSS) in which a score $\geq 4$ indicates clinically significant fatigue [13]. The scale has been recently validated in a large sample size of healthy subjects, who showed a mean score of $3.0 \pm 1.08$ [14].

We also collected the following parameters for each patient with ALS included in the study: date and area of ALS onset, medical and drug history, forced vital capacity (FVC), and use of nocturnal ventilation. Severity of physical impairment was assessed by the ALS Functional Rating Scale-Revised (ALSFRS-R) [15]. Depression was evaluated using Beck Depression Inventory (BDI) [16]. A score of $>14$ indicates depression.

This study was approved by the institutional review board of the University of Palermo. Written informed consent was obtained from all participants.

Statistical analyses were carried out using SPSS 10.0 software (SPSS, Chicago, IL, USA). Continuous variables were compared with the Mann-Whitney U test, and categorical variables were analyzed using the chisquare test. Binary correlation was evaluated with Spearman's correlation $(\rho)$. Clinical and demographic characteristics, and sleep-related complaints were also compared in patients with and without fatigue (FSS $\geq$ or $<4$ ) using a univariate statistical analysis. Results were controlled using a stepwise multiple logistic regression model. Odds ratios (ORs) and 95\% confidence intervals (CIs) were calculated. Because of multiple comparisons, a $P$ value of $<0.01$ (two-tailed) was considered significant.

\section{Results}

A total of 91 patients with ALS (36 women, 55 men; mean age: $61.26 \pm 10.1$ years) were included in the study. The site of ALS onset was bulbar in 24 patients (26.4\%). Patients had a mean duration of ALS symptoms of $24.9 \pm 15.4$ months, a mean ALSFRS-R score of $33.85 \pm 8.75$, and a mean FVC of $75.1 \% \pm 23.45$. Twelve patients used non-invasive ventilation. All but six patients were taking riluzole. Mean BDI score was $10.5 \pm 5.12$, and 25 patients $(27.5 \%)$ were depressed. Sixteen patients $(17.6 \%)$ were taking antispasticity or antidepressants. No patients had overt dementia.

The mean global PSQI score of patients with ALS was $7.0 \pm 3.74$. Fifty-two patients $(57.14 \%)$ were classified as poor sleepers (global PSQI score $>5$ ). The mean value of all components of PSQI is reported in Table 1 . The most common sleep problems reported by patients with ALS were nocturia $(62.6 \%)$, nocturnal cramps $(45 \%)$, difficulties turning in bed $(38.5 \%)$, and snoring $(36.3 \%)$. Mean ESS score was $7.48 \pm 2.54$, and 22 patients $(24.2 \%)$ had EDS.

The mean FSS score was $4.35 \pm 1.1$. Using the accepted cut-off of $\geq 4,48$ of 91 patients $(52.75 \%)$ reported clinical significant fatigue. FSS score was significantly correlated with ALSFRS-R score $(\rho=-0.51$, $P<0.001)$, FVC $(\rho=-0.34, P=0.001)$, ESS score $(\rho=0.28, \quad P=0.007), \quad$ BDI score $\quad(\rho=0.31$, $P=0.003)$, and global PSQI score $(\rho=0.25$, $P=0.01)$.

Table 2 shows the clinical and demographic characteristics of ALS patients with and without significant fatigue (FSS $\geq 4$ ). Patients with fatigue were significantly more disabled $(P<0.001)$ and more frequently reported difficulties staying asleep $(P=0.009)$ and nocturnal complaints $(P=0.002)$ than those without fatigue. In the multivariate analysis - including ALSFRS-R, FVC, ESS, BDI, global PSQI, insomnia, and night-time complaints as confounding factors - patients' disability (OR $0.91,95 \%$ CI $0.86-0.97, P=0.003$ ) and nocturnal complaints (OR 3.19, 95\% CI 1.24-8.24, $P=0.01)$ were significant predictors of fatigue.

\section{Discussion}

In this cross-sectional study, we demonstrated that fatigue, a troublesome and disabling symptom in ALS, 
is associated with sleep disturbances, in particular sleep maintenance insomnia and night-time complaints. Moreover, in common with previous findings [1-3], we confirmed that fatigue is a frequent problem in patients with ALS, and in our study, more than half of patients reported clinical significant fatigue.

Both peripheral fatigue (muscle fatigability owing to disorders of muscle and neuromuscular junction) and central fatigue (the subjective sense of fatigue essentially perceived at the level of the central nervous system) could contribute to physical fatigue in ALS [4]. Moreover, incapacitating fatigue could be the result of a potentially correctable sleep-related abnormality [4]. In our study, sleep maintenance insomnia was significantly correlated with the presence of fatigue, and night-time complaints strongly predicted clinical significant fatigue after physical disability had been controlled for. Our results, then, strengthen the hypothesis that sleep disturbances could be an important cause of fatigue in patients with ALS. As nocturia and muscle cramps were the most frequently reported night-time complaints, it is possible that addressing these symptoms may improve not only sleep but also fatigue in many patients. On the

Table 1 Components PSQI scores in patients with ALS

\begin{tabular}{ll}
\hline Components of PSQI & ALS group, mean \pm SD \\
\hline Subjective sleep quality & $0.93 \pm 0.74$ \\
Sleep latency & $1.32 \pm 1.11$ \\
Sleep duration & $1.14 \pm 0.9$ \\
Habitual sleep efficiency & $1.07 \pm 0.92$ \\
Sleep disturbances & $1.33 \pm 0.61$ \\
Use of sleeping medication & $0.32 \pm 0.84$ \\
Daytime dysfunction & $1.0 \pm 0.86$ \\
Global PSQI score & $7.0 \pm 3.74$ \\
\hline
\end{tabular}

ALS, amyotrophic lateral sclerosis; PSQI, Pittsburgh Sleep Quality Index. contrary, initial insomnia and unrefreshing sleep were not significantly associated with the presence of fatigue.

These findings have implications for clinical management. As data on pharmacological agents relieving fatigue in ALS are sparse, the clinician must use a multimodality approach. It has been shown that psychostimulant treatment (modafinil) could be helpful, if no treatable cause is identified [17]. Treatment of associated depression or poor nutrition could also be effective. On the other hand, because there is some evidence that ventilatory support could reduce fatigue in other populations, non-invasive ventilation should be considered in those patients with respiratory impairment. In addition, muscle cramps should be aggressively treated, and although at present there are insufficient data to support or refute any specific intervention, gabapentin, vitamin E, and quinine could be of use [18]. Nocturia can be treated with a number of possible therapy options, depending on the etiology of the condition, including anticholinergics (e.g. tolterodine, darifenacin, solifenacin), $\alpha$-blockers/ $\alpha$-adrenergic blockers (e.g. tamsulosin), or with desmopressin [19]. Finally, a variety of medications are available for use in patients with insomnia, including benzodiazepines, the non-benzodiazepine hypnotics (zolpidem, eszopiclone, and zaleplon), antihistamines, and the sedating antidepressants (trazodone, amitriptyline, and mirtazapine).

Another interesting finding of the study was that we documented a fair-to-moderate association between fatigue and depression, daytime somnolence, and respiratory function. These associations however were no longer significant when other contributing factors, such as physical impairment, were accounted for, showing that ALS severity was the most important clinical predictor of fatigue in the patients studied. Similarly, McElhiney et al. [3] also found an association

Table 2 Characteristics of patients with ALS with and without clinical significant fatigue

\begin{tabular}{llll}
\hline & Patients with fatigue $(n=48)$ & Patients without fatigue $(n=43)$ & $P$ \\
\hline Age, years & $62.6 \pm 8.66$ & $59.77 \pm 11.47$ & n.s. \\
Gender, M/F & $29 / 19$ & $26 / 17$ & n.s. \\
Duration of ALS symptoms, months & $23.46 \pm 14.78$ & $26.51 \pm 16.02$ & n.s. \\
Bulbar ALS onset & $10(20.8 \%)$ & $14(32.6 \%)$ & n.s. \\
FVC, \% of predicted & $71.02 \pm 21.87$ & $79.65 \pm 24.55$ & n.s. \\
ALSFRS-R score & $30.65 \pm 8.59$ & $37.24 \pm 7.54$ & $<0.001$ \\
ESS score & $7.81 \pm 2.74$ & $7.12 \pm 2.26$ & n.s. \\
BDI score & $10.69 \pm 4.83$ & $10.28 \pm 5.47$ & n.s. \\
Global PSQI score & $7.36 \pm 3.72$ & $6.67 \pm 3.75$ & n.s. \\
Difficulties falling asleep & $17(35.4 \%)$ & $17(39.5 \%)$ & n.s. \\
Difficulties staying asleep & $31(64.6 \%)$ & $16(37.2 \%)$ & 0.009 \\
Unrefreshing sleep & $18(37.5 \%)$ & $10(23.3 \%)$ & n.s. \\
Nocturnal complaints & $28(58.3 \%)$ & $11(25.6 \%)$ & 0.002 \\
\hline
\end{tabular}

ALS, amyotrophic lateral sclerosis; FVC, forced vital capacity; ALSFRS-R, ALS Functional Rating Scale-Revised; ESS, Epworth Sleepiness Scale; BDI, Beck Depression Inventory; PSQI, Pittsburgh Sleep Quality Index. 
between clinical significant fatigue and ALS severity. They reported that the later stage of illness was associated with higher rates of fatigue, and there was some degree of overlap between symptoms of fatigue and depression. In partial contrast, Ramirez et al. [2] reported that fatigue was greater in the youngest patients and worsened during follow-up, even if there was no correlation between fatigue and ALSFRS-R score. Lou et al. [1] also studied a small population of 25 patients and found that fatigue had a significant impact on quality of life, but the severity of fatigue did not correlate with physical impairment, measured using the ALSFRS. Taken together, these studies show that fatigue can have a great impact on patients with ALS, although differences in the populations studied and in the methodologies used might account for the disparities reported.

The main limitation of the present study is the lack of objective measures of sleep quality, such as actigraphy or polysomnography, to verify the accuracy of selfreport data, but we think it is extremely difficult to obtain these measures when examining large populations of patients. Moreover, the tools used in this study are brief and easily administered, allowing a simple and straightforward implementation in everyday clinical practice.

Another limitation of the study is that we used a unidimensional instrument to assess fatigue, so we could not measure physical and mental fatigue independently.

In conclusion, the results of our study further strengthen the need for routine enquiries about fatigue in ALS and suggest that questions about sleep and nocturnal complaints should be included in the regular review of patients with ALS, in particular those reporting fatigue.

\section{Disclosure of conflict of interest}

The authors report no financial support and no conflicts of interest.

\section{References}

1. Lou JS, Reeves A, Benice T, Sexton G. Fatigue and depression are associated with poor quality of life in ALS. Neurology 2003; 60: 122-123.

2. Ramirez C, Piemonte ME, Callegaro D, Da Silva HC. Fatigue in amyotrophic lateral sclerosis: frequency and associated factors. Amyotroph Lateral Scler 2008; 9: 7580.
3. McElhiney MC, Rabkin JG, Gordon PH, Goetz R, Mitsumoto $\mathrm{H}$. Prevalence of fatigue and depression in ALS patients and change over time. J Neurol Neurosurg Psychiatry 2009; 80: 1146-1149.

4. Lou JS. Fatigue in amyotrophic lateral sclerosis. Phys Med Rehabil Clin N Am 2008; 19: 533-543.

5. Maessen M, Veldink JH, Onwuteaka-Philipsen BD, et al. Trends and determinants of end-of-life practices in ALS in the Netherlands. Neurology 2009; 73: 954-961.

6. Ferguson KA, Strong MJ, Ahmad D, George CF. Sleepdisordered breathing in amyotrophic lateral sclerosis. Chest 1996; 110: 664-669.

7. Lo Coco D, Mattaliano P, Spataro R, Mattaliano A, La Bella V. Sleep-wake disturbances in patients with amyotrophic lateral sclerosis. J Neurol Neurosurg Psychiatry 2011; 82: 839-842.

8. Lo Coco D, Piccoli F, La Bella V. Restless legs syndrome in patients with amyotrophic lateral sclerosis. Mov Disord 2010; 25: 2658-2661.

9. Limousin N, Blasco H, Corcia P, Arnulf I, Praline J. The high frequency of restless legs syndrome in patients with amyotrophic lateral sclerosis. Amyotroph Lateral Scler 2011; 12: 303-306.

10. Brooks BR, Miller RG, Swash M, et al. El Escorial revisited: revised criteria for the diagnosis of amyotrophic lateral sclerosis. Amyotroph Lateral Scler Other Motor Neuron Disord 2000; 1: 293-299.

11. Busse DJ, Reynolds CF III, Monk TH, et al. The Pittsburgh Sleep Quality Index: a new instrument for psychiatric practice and research. Psychiat Res 1989; 28: 193-213.

12. Johns MW. A new method for measuring daytime sleepiness: the Epworth sleepiness scale. Sleep 1991; 14: 540545.

13. Krupp LB, LaRocca NG, Muir-Nash J, Steinberg AD. The fatigue severity scale. Application to patients with multiple sclerosis and systemic lupus erythematosus. Arch Neurol 1989; 46: 1121-1123.

14. Valko PO, Bassetti CL, Bloch KE, Held U, Baumann CR. Validation of the fatigue severity scale in a Swiss cohort. Sleep 2008; 31: 1601-1607.

15. Jesse M, Cedarbaum JM, Stambler N, et al. The ALSFRS-R: a revised ALS functional rating scale that incorporates assessments of respiratory function. J Neurol Sci 1999; 169: 13-21.

16. Beck A, Ward $\mathrm{CH}$, Mendelson $\mathrm{M}$, et al. An inventory for measuring depression. Arch Gen Psychiatry 1961; 4: 561571.

17. Rabkin JG, Gordon P, McElhiney M, et al. Modafinil treatment of fatigue in patients with ALS: a placebocontrolled study. Muscle Nerve 2009; 39: 297-303.

18. Miller RG, Jackson CE, Kasarskis EJ, et al. Practice parameter update: the care of the patient with amyotrophic lateral sclerosis: multidisciplinary care, symptom management, and cognitive/behavioral impairment (an evidence-based review): report of the Quality Standards Subcommittee of the American Academy of Neurology. Neurology 2009; 73: 1227-1233.

19. Ancoli-Israel S, Bliwise DL, Nørgaard JP. The effect of nocturia on sleep. Sleep Med Rev 2011; 15: 91-97. 